\title{
Performance of the missing transverse energy measurement with the ATLAS detector in commissioning data
}

\section{Pavel WEBER ${ }^{* \dagger}$}

Kirchhoff Institut für Physik, Universität Heidelberg,

E-mail: pavel@kip.uni-heidelberg.de

\begin{abstract}
The measurement of the missing transverse momentum in events produced in proton- proton collisions is an important ingredient in the physics program of the LHC. In 2008 the ATLAS detector has been operated to commission and integrate the individual sub-detectors and a large data-set of more than 200 millions of cosmic and randomly triggered events has been recorded. It has been shown that the noise in more than 200000 cells in the ATLAS calorimeter does not introduce a significant bias in the measurement of the scalar and vectorial transverse energy. Moreover, the noise in the ATLAS liquid argon calorimeter is compatible with the expectation for uncorrelated Gaussian noise. Also large energy deposits of several TeV in the ATLAS calorimeters have been analyzed. It is shown that the transverse momentum spectrum of these high energy depositions can be explained by photons radiated from high energetic muons produced in cosmic rays. Furthermore, it is investigated how energy depositions induced by cosmic rays can be rejected based on jet properties. This study shows that ATLAS is well prepared to analyze first collision data expected in late 2009 .
\end{abstract}

European Physical Society Europhysics Conference on High Energy Physics

July 16-22, 2009

Krakow, Poland

*Speaker.

${ }^{\dagger}$ on behalf of the ATLAS Collaboration. 


\section{Introduction}

The ATLAS detector [1] is designed to exploit the full discovery potential of the Large Hadron Collider (LHC). Many signals in the ATLAS search program, e.g. SUSY, extra dimensions, miniblack holes, are characterized by a large missing transverse energy. Therefore, a good measurement of missing transverse energy $E_{T}^{\text {miss }}$ is very important for successful searches for new physics. Over 200 millions of cosmic events recorded during the detector commissioning phase in SeptemberOctober 2008 are used to estimate the performance of the $E_{T}^{\text {miss }}$ measurement in ATLAS and to understand the calorimeter noise, cosmic ray background, fake $E_{T}^{\text {miss }}$ and rejection cuts.

\section{Missing $E_{T}$ measurement and reconstruction}

The calculation of $E_{T}^{\text {miss }}$ is primarily based on the measurement of the energy depositions in cells of the ATLAS calorimeter. The additional corrections to $E_{T}^{\text {miss }}$ come from muon momenta measured by the muon spectrometer and energy losses in the Liquid Argon (LAr) cryostat. Only the calorimetric contribution to $E_{T}^{\text {miss }}$ with random trigger and cosmic event data is studied and considered in this note. The calorimeter input to the $E_{T}^{\text {miss }}$ reconstruction is formed either from the cells or from so-called topoclusters depending on the noise suppression method: cell-based or topocluster-based. The first method uses all cells with energies above $2 \sigma_{\text {noise }}$, where $\sigma_{\text {noise }}$ is the standard deviation of the cell noise distribution. The topocluster-based approach is based on 3-dimensional topological calorimeter clusters which are formed around a seed cells [2]. The cells remaining after the application of the noise suppression algorithms are used for the $E_{T}^{\text {miss }}$ calculation and calibration. A detailed description of the $E_{T}^{\text {miss }}$ reconstruction is given in [3].

\section{Missing $E_{T}$ measurement performance in random trigger data}

The results presented in this section are based on data taken in 35 runs between September $10^{\text {th }}$ and October $23^{\text {rd }}$ 2008. The events collected with these runs are "randomly triggered" i.e. they do not contain any real energy depositions in the calorimeter system, only the electronic noise. All variables are calculated at the electromagnetic scale, as no hadronic calibration has been applied.

In Figure 1 the $E_{T}^{\text {miss }}$ distribution from

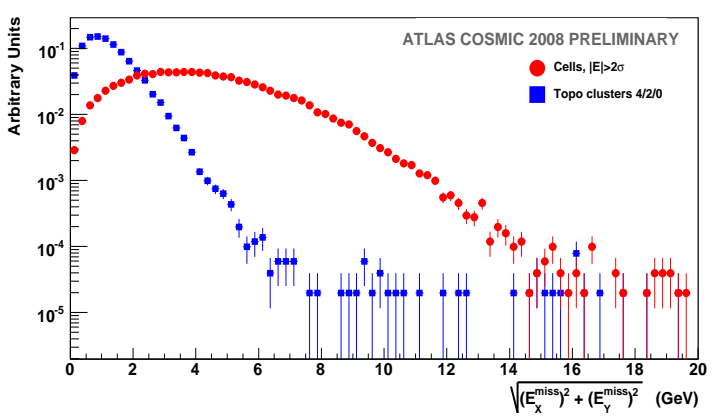

Figure 1: Inclusive $E_{T}^{\text {miss }}$ distribution with cell-based and topocluster-based suppression method. run taken on October $14^{\text {th }} 2008$ for both the cell-based and topocluster-based noise suppression is plotted. It shows that a better noise suppression is provided by the topocluster-based method. Tails in the distribution ( beyond $8 \mathrm{GeV}$ for topocluster-based and $16 \mathrm{GeV}$ for cell-based methods), contributing less than $0.1 \%$ of events, come from coherent noise in a specific region of the LAr presampler. This problem has since been fixed. In order to check the stability of the noise, the $E_{T}^{\text {miss }}$ distribution is reconstructed for each 

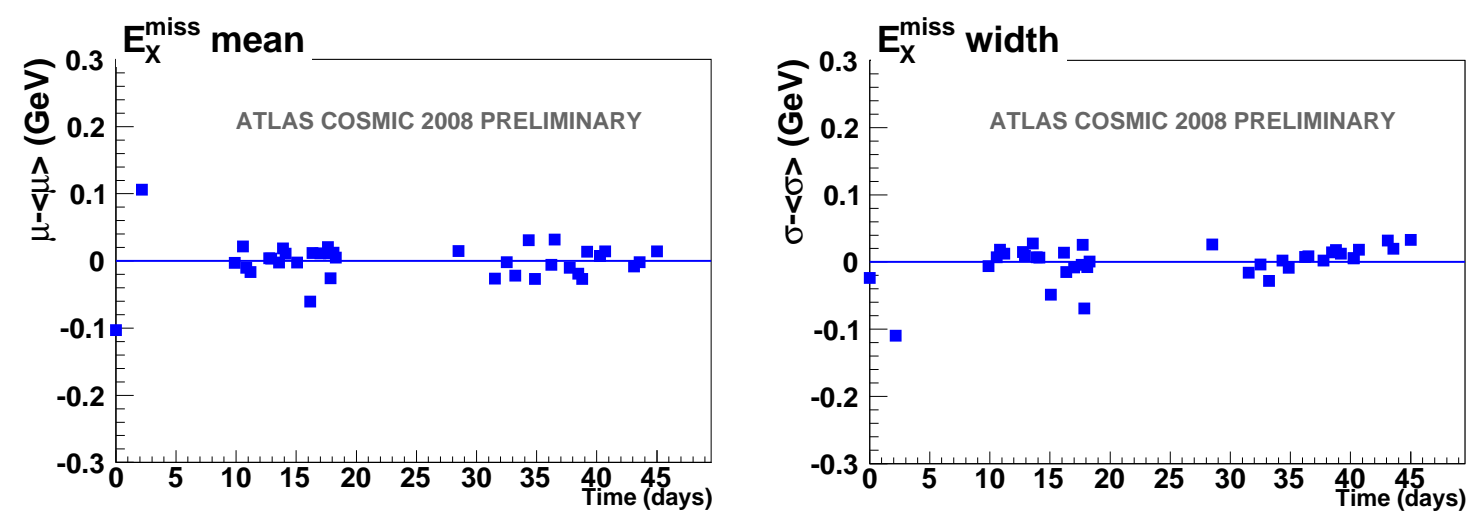

Figure 2: Deviation of the mean of the $E_{x}^{\text {miss }}$ distribution from its average value $\langle\mu\rangle$ (left) and deviation of the width of the $E_{x}^{\text {miss }}$ distribution from its average value $\langle\sigma\rangle$ over period of the month and a half.

run, seperately, using the standard topocluster algorithm; the distributions are fitted with a Gaussian and the mean $\mu$ and standard deviation $\sigma$ are extracted. Figure 2 shows the results as function of time. Only small deviations from the average are observed demonstrating the stability of the $E_{T}^{\text {miss }}$ measurement.

\section{Missing $E_{T}$ in cosmic data and cleaning cuts}

The analysis presented in this section is based on cosmic data collected in a run taken in September 2008. In this run cosmic muons depositing sufficiently large energy in the calorimeter were triggered by the Level-1 Calorimeter Trigger. The collected data are compared with cosmic ray Monte Carlo samples which were produced using the known cosmic muon flux at ground level [4]. In Figure 3 (left) the Jet EM Fraction (EMF) is shown. The EMF is defined as the ratio of the energy deposited in the EM calorimeter (Presampler, EM Liquid Argon, and the 1st layer of the Forward Calorimeter) and the energy seen in the whole calorimeter. Figure 3 (left) shows that the
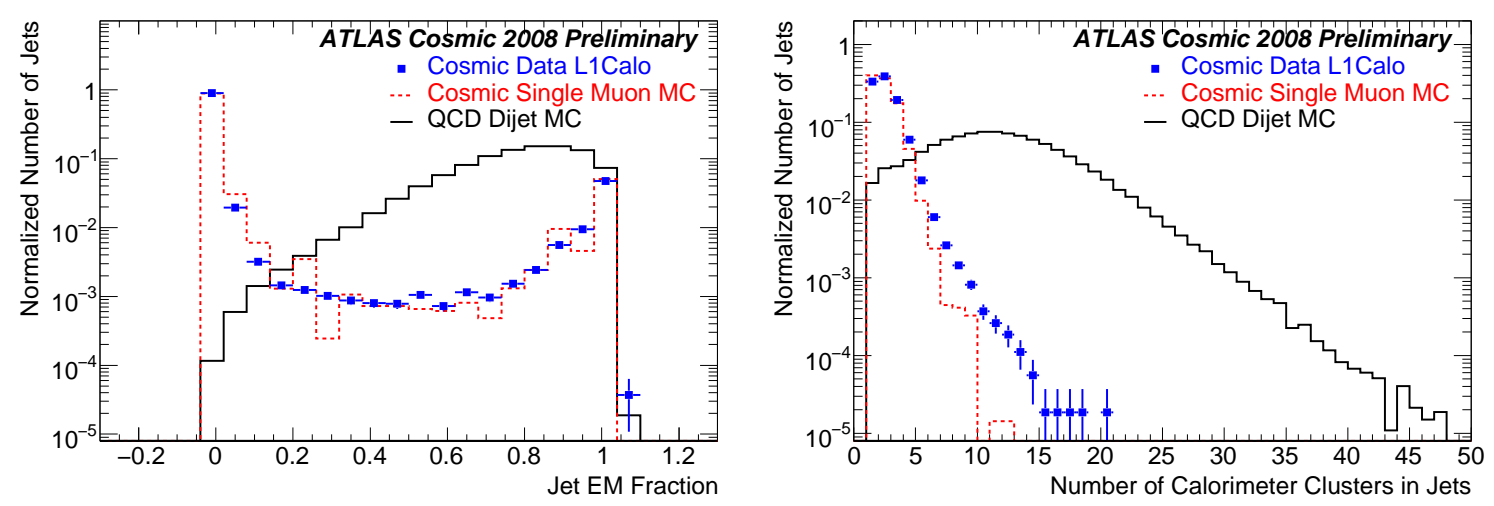

Figure 3: The 2008 cosmic ray data compared to a cosmic ray Monte Carlo simulation and QCD d-jet Monte Carlo sample in terms of the jet electromagnetic fraction (left) and the number of calorimeter clusters (right). 
EMF can be used to effectively reject fake jets coming from cosmic muons as QCD jets generally show up at larger values of the EMF. As an alternative the number of calorimeter clusters found in a jet can be used as shown in Figure 3 (right). The distribution of the jet $E_{T}$ after cleaning cuts based on the methods described is shown in Figure 4.

\section{Conclusion}

The presented results from random triggered and cosmic events show a good performance of transverse energy measurement and reconstruction. They also give a first evidence that the measurement is stable in time, demonstrating the robustness of the calorimeter. The most effective calorimeter noise suppression is provided by the topocluster-based method, which shows a better resolution than the cell-based noise suppression. Moreover, the cleaning cuts studied provide good separation between real QCD jets and fake jets from cosmics. The good agreement of the transverse momentum spectrum obtained in the cosmic

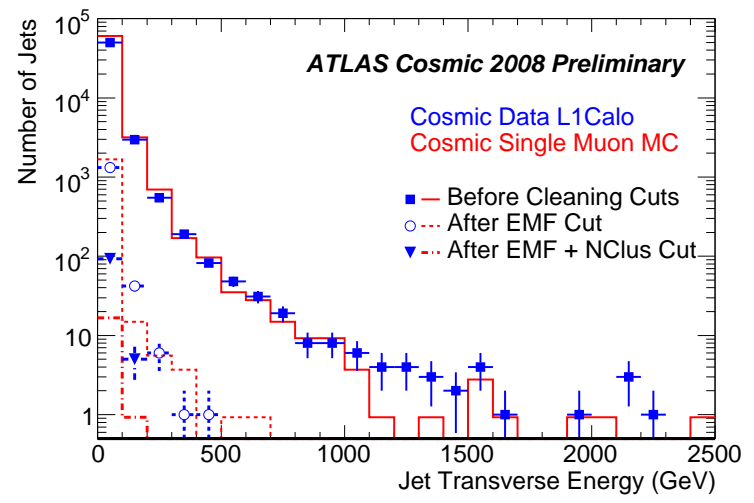

Figure 4: The distribution of the jet transverse energy from cosmic data before cleaning cuts (blue squares) after electromagnetic fraction cut (empty circles) and after combined cut based on electromagnetic fraction and number of calorimeter clusters. data with the expectations demonstrate our advanced understanding of the ATLAS calorimeter system.

\section{References}

[1] G. Aad et al. [ATLAS Collaboration], JINST 3 (2008) S08003.

[2] Cojocaru, $\mathrm{C}$ et al. Hadronic calibration of the ATLAS liquid argon end-cap calorimeter in the pseudorapidity region 1.6-1.8 in beam tests, NIM, A531 (2004) 481-514

[3] G. Aad et al. [The ATLAS Collaboration], arXiv:0901.0512 [hep-ex].

[4] A. Dar, Atmospheric Neutrinos, Astrophysical Neutrons, and Proton-Decay Experiments Phys. Rev. Letters 51, 227 (1983) 